\title{
Conflits Autour De L'exploitation Halieutique Du Fleuve Comoé Et De Ses Affluents
}

\author{
Aka Koffi Sosthène \\ Docteur en géographie, Attaché de recherche au Centre de Recherches \\ Océanologiques (C.R.O), Treichville, Abidjan-Côte d'Ivoire
}

doi: 10.19044/esj.2017.v13n5p317URL:http://dx.doi.org/10.19044/esj.2017.v13n5p317

\begin{abstract}
The purpose of this article is to identify the conflicts arising from the exploitation of the Comoé River. Côte d'Ivoire has a dense hydrography which is distinguished by four main rivers of which the Comoé River (1160 $\mathrm{km}$ ) is one of them. This river area, which has always been exploited by the various villages bordering it, is perceived by the latter as their property. Thus, they ensure customary management in accordance with marked territories. The immense benefits of this river to village communities sometimes give rise to tensions and conflicts. What are the causes of these different types of conflicts with regards to the Comoé River? In other words, what are the conditions for their gestation?

The objective of this study is to reveal the conflicts surrounding the fisheries exploitation of the Comoé River and to propose solutions for a sustainable fishery. This study is based on desk research and land surveys.

It appears from the above that the exploitation of the aquatic area of the Comoé River generates sporadic conflicts. These conflicts arise mainly because of a poor implementation of the clauses of the contract between indigenous and non-native fishers, dominated by the monthly fee and the unhealthy practices of the actors. The management of these oppositions of interests is ensured by a traditional regulation, coupled with that of the administration when the situation is of great magnitude.
\end{abstract}

Keywords: Côte d'Ivoire, Comoé river, conflicts, fisheries exploitation

\section{Resume}

L'objet de cet article est d'identifier les conflits issus de l'exploitation du fleuve Comoé. La Côte d'Ivoire dispose d'une hydrographie dense dans laquelle se distinguent quatre principaux fleuves dont le Comoé $(1160 \mathrm{~km})$. Cet espace fluvial, exploité depuis toujours par les différents villages qui le bordent est perçu par ceux-ci comme leur 
propriété. Ainsi, ils assurent la gestion coutumière conformément à des territoires marqués. De ce fait, les multiples usages qui en sont faits par les communautés villageoises donnent lieu quelquefois à des tensions et conflits. Quelles sont les causes des différents types de conflits sur le Comoé ? En d'autres termes, quelles sont les conditions de leur gestation?

Cette étude a pour objectif de révéler les conflits autour de l'exploitation halieutique du fleuve Comoé et de proposer des solutions pour une pêche durable.

La présente étude s’appuie sur une recherche documentaire et les enquêtes de terrains.

Il apparaît au regard de ce qui précède que l'exploitation de l'espace aquatique du Comoé génère des conflits sporadiques. Ils surviennent surtout pour une mauvaise mise en pratique des clauses du contrat entre autochtones et pêcheurs allogènes, dominées par la redevance mensuelle et les pratiques malsaines des acteurs. La gestion de ces oppositions d’intérêts est assurée par une réglementation traditionnelle, couplée toutefois à celle de l'administration lorsque la situation est d'une grande ampleur.

Mots-clés : Côte d’Ivoire, fleuve Comoé, conflits, exploitation halieutique

\section{Introduction et justification de l'etude}

Les conflits autour des plans d'eau et des terres font partie aujourd'hui des problématiques géographiques les plus remarquables. Cette situation est favorisée par l’évolution démographique. Le continent africain connaît depuis des décennies des foyers de tensions épars, basés sur des intérêts. Qu'il s’agisse des ressources du sous-sol ou des grands espaces aquatiques, leurs différentes formes d'exploitation sont des sources de divergences. Il peut y avoir à cet effet "des conflits ouverts actuels ou chroniques et être susceptibles de déboucher sur d'autres formes de conflits dans les années à venir" (Merlet, 2011, p.10). Les conflits se définissent comme des oppositions d'intérêts, d'opinions entre des États ou des individus. La théorie de Norem (1977) les organise en quatre groupes que sont les conflits relationnels, les conflits structurels, les conflits d'intérêts et les conflits de valeurs. Le type de conflits qui sied à l'étude est celui d'intérêts, car il se produit en cas d'incompatibilité entre les besoins perçus et les besoins réels. Ce type de conflit surgit souvent, suite à une incompréhension entre les gestionnaires des espaces et les utilisateurs. D’emblée, retenons que les droits de gestion des terres et des espaces aquatiques en Afrique sont des droits de transfert pour la plupart (héritage, legs). Á cet égard, les détenteurs sont des individus ou des communautés. Ainsi, "les terres comme les bordures des plans d'eau sont souvent habitées et exploitées depuis des générations, mais les habitants ne disposent pas de 
documents formels attestant leurs droits" (Merlet, 2011, p.27) parce qu'ils sont analphabètes pour la plupart. Et pourtant, il existe des textes sur l'utilisation des bordures des eaux et la navigation.

En Côte d'Ivoire, l'espace aquatique et ses ressources font partie du patrimoine de l'État qui en détient le droit de pêche. Les eaux continentales sont souvent régies par des arrêtés interministériels, ministériels, ou encore des circulaires. En la matière, le plus important qui soit en rapport avec les fleuves date de 1984 (Arrêté interministériel n001 du 8 octobre 1984) et porte sur l'institution d'un permis pour la pêche professionnelle dans les eaux intérieures. En son article 12, il stipule que les préfets et sous-préfets sont chargés de son application.

Á travers les eaux intérieures de façon générale, "la pêche continentale offre ainsi la configuration la plus complexe avec une juxtaposition de communautés de pêches autochtones et de communautés de pêches étrangères qui entretiennent trop souvent des relations conflictuelles" (FAO, 2008, p.1.). Le Comoé dispose d'un potentiel halieutique notable. Les différentes espèces de poissons de son bassin versant s'évaluent à 76, constituées en 22 familles dont 11 espèces marines selon les études menées par Yao et al. (2005). L’on note cependant la présence d'une espèce importée (Hétérotis niloticus) et d'une espèce hybride (Tilapia guinéensis et Tilapia zillii). Les autres espèces se caractérisent par la présence de Mormydae et des Mochokidae. De ce fait, ce plan d'eau est soumis à une exploitation accrue par les populations d'origines diverses. L'espace halieutique du Comoé connaît des tensions quoique relatives d'un espace de pêche à un autre, avec divers motifs. Quelles sont les causes des différents types de conflits sur le Comoé ? En d'autres termes, quelles sont les conditions de leur gestation?

Cette étude ayant pour cible le Comoé et ses affluents a pour objectif de révéler les conflits autour de l'exploitation halieutique du fleuve Comoé et de proposer des solutions pour une pêche durable. Elle s’appuie sur une méthodologie de recherche.

\section{Methodologie de la recherche}

L’approche méthodologique utilisée pour atteindre les objectifs repose sur une recherche documentaire et des enquêtes de terrain. Au niveau du premier volet, les publications traitant les différents aspects des problèmes fonciers et les conflits sur les plans d'eau ont été exploitées. Les articles, les documents, les ouvrages de la FAO, les rapports de séminaires, de colloques, ont été d'un apport utile. Les écrits de Merlet (2011) et de Cormier-Salem (2000) abordant respectivement "les aspects liés au foncier et au droit de l'eau" et au mode "d’appropriation des ressources" ont été essentiels pour cerner les contours du sujet. Aussi, se révèle-t-il que cette 
réalité conflictuelle est vécue dans la quasi-totalité des pays africains et aboutit souvent à une équation tridimensionnelle de conflit-préventionrésolution dans des aires géographiques bien définies et qui constituent le support physique, objet d’interprétation. L’enquête de terrain, quant à elle, privilégie le guide d'entretien pour interviewer les acteurs: les autorités administratives et coutumières, les responsables des services de pêche, les pêcheurs des localités visitées. L’appui des responsables départementaux, régionaux de pêche, des préfets et sous-préfets qui ont en charge la gestion des activités halieutiques sur le Comoé a permis de localiser les foyers de tensions. En outre, tous les villages qui le bordent ont été parcourus et les aspects se rapportant aux différents modes de gestion de l'espace aquatique et aux conflits ont été abordés singulièrement. Cette approche a permis de dénombrer les pêcheurs, de cerner les différents systèmes de gestion coutumière de chaque localité et d’identifier les causes des conflits. Par ailleurs, un accent particulier a été mis sur la compréhension des types de relations existant entre l'administration, les acteurs et les autochtones riverains. Des localités cibles de conflits ont donc été identifiées : Moossou, Abrotchi, Nianda, Yakassé-Comoé, Niablé, M’Basso Agni et M’Basso Attié.

Les informations recueillies ont été traitées, analysées et révisées de commun accord avec les enquêtés. Aussi, une restitution était-t-elle faite aux différentes parties avant de quitter un espace de tension pour un autre. Cet article s’articule autour de 3 principaux points :

- $\quad$ Présentation de la zone d’étude et des systèmes de gestion mis en place.

- $\quad$ Types de conflits, causes et moyens de règlement.

- $\quad$ Analyse des moyens de réglementation des conflits et esquisse de solutions pour une gestion durable de la pêche sur le Comoé et ses affluents.

\section{I/ Presentation de la zone d'etude et des systemes de gestion mis en place}

L'espace aquatique est par excellence un facteur de progrès économique compte tenu des activités développées et des emplois annexes induits. En effet, la présence d'un lac, d'un cours d'eau dans une région est importante pour le développement de diverses activités dont la pêche. Le Comoé n’échappe pas à l'empreinte des pêcheurs. Comment se présente cet espace aquatique à travers son bassin versant ? Comment les populations autochtones le perçoivent-ils ? Quel est le type de gestion adoptée ?

\section{1-Présentation de la zone d'étude.}

Le Comoé (1160 km) est le plus long des fleuves de Côte d'Ivoire. Il dispose d'un bassin versant de $75000 \mathrm{~km}^{2}$, à cheval sur les pays frontaliers, notamment le Ghana, le Mali, et le Burkina Faso. Il prend sa source au Burkina Faso à partir de deux principaux affluents que sont le Comoé dans la 
région de Banfora et la Léraba à Sindou. Ces deux cours d'eau se combinent à la frontière ivoirienne pour donner naissance au grand fleuve Comoé dont la partie ivoirienne est longue de $900 \mathrm{~km}$. Á l'image de tous les grands fleuves ivoiriens, le Comoé a une capacité renforcée par ses nombreux affluents, indispensables pour la pêche, surtout en période de crue, dont les plus connus au nombre de 9, du nord au sud : Kolonkoko, Bouin, Inrigou, Kongo, Segbono, Baya, Ba, Bêki, Manzan. Les figures 1 et 2 indiquent respectivement sa localisation et son bassin versant. Toutes ces branches concourent à le rendre puissant confirmé par son débit qui subit aussi la variation pluviométrique. Il se jette dans la lagune Ebrié où son embouchure constamment obstruée fait une particularité par rapport aux autres grands fleuves du pays.

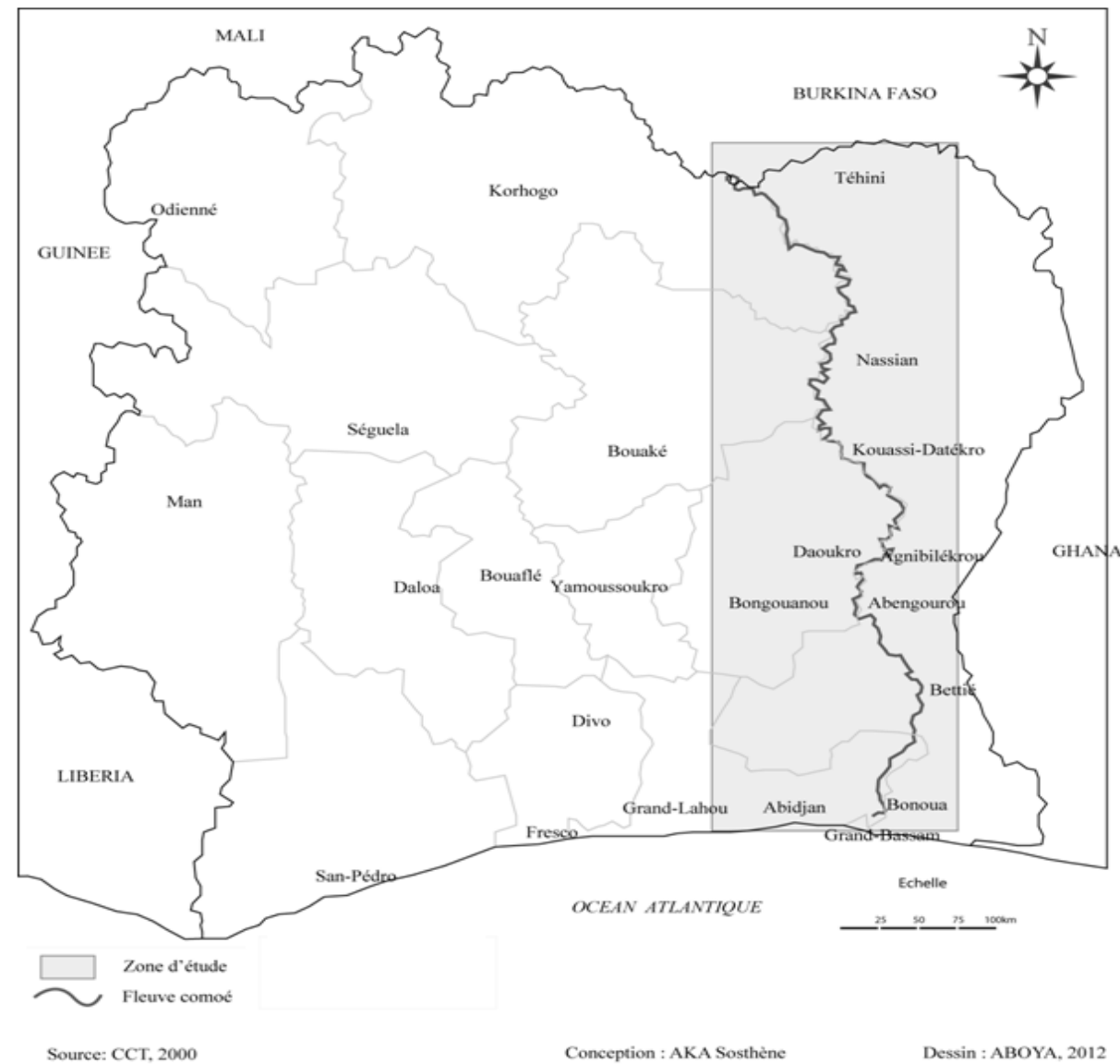

Figure 1 : Localisation de l'espace d'étude 


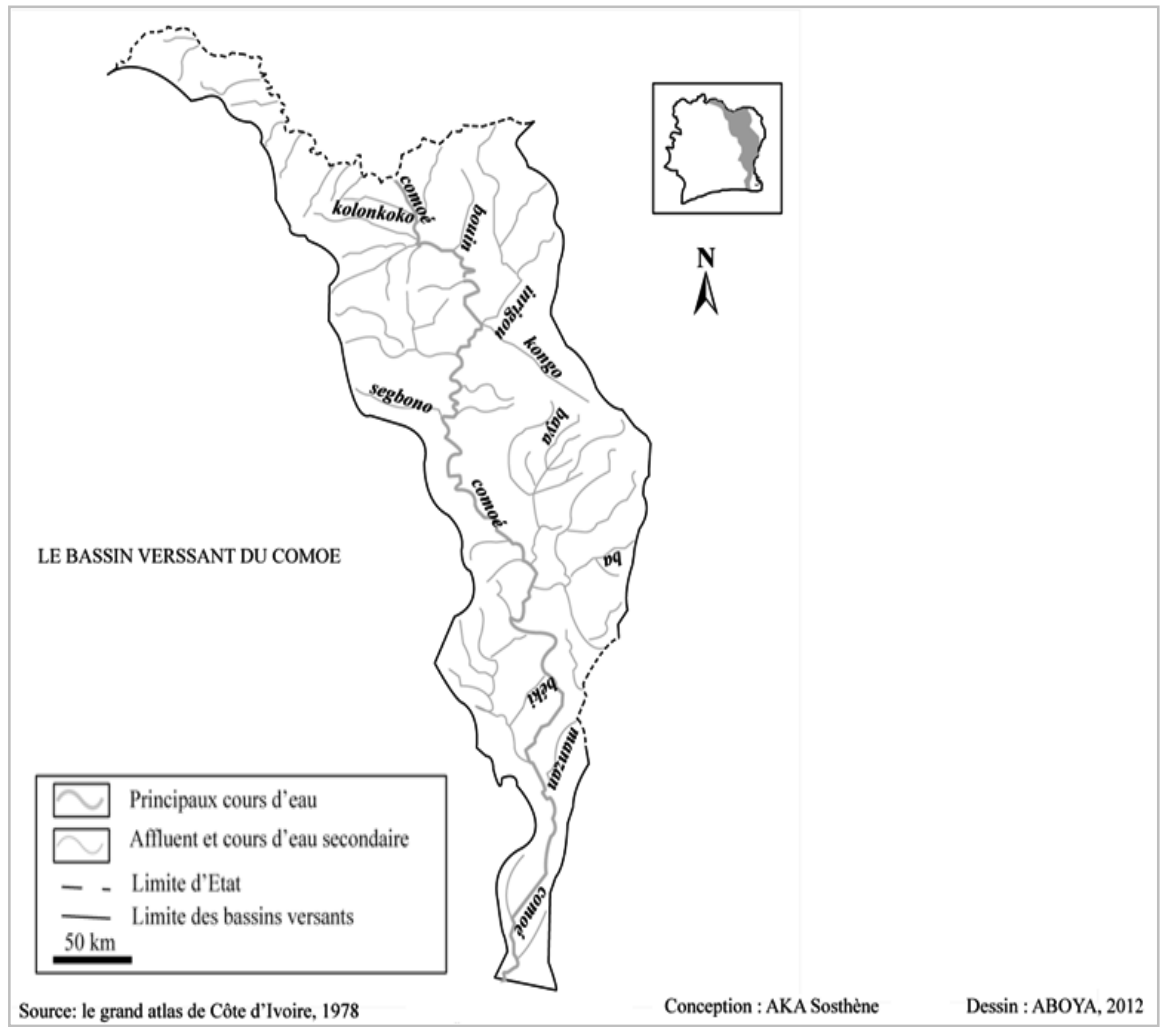

Figure 2 : Bassin versant du Comoé

\section{2-Mode de gestion ou d'exploitation du fleuve Comoé}

Les eaux ivoiriennes sont confrontées souvent à deux types de gestion: la gestion moderne régie par des textes, des lois et la gestion traditionnelle, l'œuvre des populations autochtones riveraines.

Le mode de gestion des eaux intérieures selon les textes

Le premier texte date du 24 août 1959 (loi n59-111 du 24 août 1959) et la dernière du 2 décembre 2005 (loi n²005-556 du 2 décembre 2005). Ces textes s'appliquent certes à toutes les eaux sur le territoire ivoirien, mais en particulier à la lagune et à la mer. Le volet continental est pris en compte de façon superficielle. Seul l'arrêté interministériel nº01 du 8 octobre 1984 porte institution d'un permis pour la pêche professionnelle dans les eaux intérieures. L'article premier souligne que, seuls les titulaires d'un permis auront accès aux eaux. Les articles 4 et 5 , quant à eux, indiquent respectivement les pièces à fournir pour son obtention, et la fixation d'une plaque d'immatriculation. Les sous-préfets et préfets sont chargés de son application (article 12). L'arrêté ministériel $n^{\circ} 087 / \mathrm{HDR} / \mathrm{DP}$ du 4 février 1985, quant à lui porte sur la fixation des taxes relatives à l'exercice de la pêche professionnelle sur les eaux intérieures du domaine public ; tandis que 
la décision $\mathrm{n}^{\circ} 105$ Minefor/CAB/DPN-PPC-EP du 2 septembre 1988 se penche sur la qualité des engins, les dimensions des mailles réglementées à $35 \mathrm{~mm}$ et plus. La dynamique dans l'application des lois constitue un énorme problème. La réelle difficulté au niveau de la pêche vient des attributions. Les agents des services halieutiques sont des civils et n'arrivent pas à s'imposer sur le terrain. Dans l'ensemble, les eaux continentales n'ont pas connu une réelle politique de pêche, encore moins dans la gestion ; excepté les lacs issus de barrages hydro-électriques et agropastoraux. Sur le fleuve Comoé, le Ministère des Ressources Animales et Halieutiques agit à partir des représentations des services de pêche dont les agents résident dans les chefs-lieux de département. Ne disposant pas de moyens adéquats pour accomplir le travail, leur rôle se limite à la sensibilisation des pêcheurs pour œuvrer dans la légalité. Ils sont souvent appuyés par les préfets et souspréfets. Malheureusement sur un effectif de 1543 pêcheurs, seuls 54 détiennent des licences (Aka, 2012). L’activité peut être qualifiée de clandestine. Les budgets souvent alloués aux différentes directions régionales et départementales leur parviennent tardivement et rend difficile la tâche à assumer. Une forme de gestion coutumière très animée rime avec cette gestion administrative quasi-ignorée des pêcheurs.

\section{3-Le mode de gestion traditionnelle}

Le mode de gestion traditionnelle se résume aux conditions d'accès aux espaces aquatiques et d'exercice de l'activité de pêche. Sur le Comoé, chaque village riverain se réclame propriétaire d'un espace fluvial qui correspond à l'étendue de son territoire et y applique ses lois coutumières. Au demeurant, Brunet et al. (1992) définissent la notion de territoire en géographie comme étant "la projection sur un espace donné des structures spécifiques d'un groupe humain, qui influe sur le mode de découpage, d'aménagement et de gestion de cet espace". Ainsi "les zones de pêche qui prolongent les terroirs fonciers constituent des espaces délimités, appropriés, contrôlés par la communauté et gérés collectivement" (Adam, 2008, p.30). Cependant, en période de décrue au niveau des affluents, il existe des biefs auxquels les populations attribuent des noms en fonction de la proximité des exploitations agricoles, qui deviennent pour la circonstance des propriétés temporaires.

Le droit coutumier se renforce. Dans la majorité des villages visités, tout pêcheur étranger à la communauté doit se faire identifier par les autorités villageoises, avoir un tuteur originaire du village pour un suivi de ses populations halieutiques, offrir une boisson forte pour sceller le contrat de pêche, prendre obligatoirement part au sacrifice annuel de mouton. Il lui est également demandé de fournir quelques belles pièces aux gestionnaires de l'eau et participer aux activités du village en cas de réalisation de travaux 
d'utilité publique (écoles, dispensaires,...), d'évènements heureux (accueil d'une autorité) ou malheureux (décès). Á cela s'ajoutent le respect des interdits et le paiement d'une redevance mensuelle, élément fondamental du contrat qui évolue de $1000 \mathrm{~F}$ à 5000 FCFA. Tout bien considéré, les chefs de cantons, les chefs de villages et les différentes notabilités associées sont les vrais gestionnaires des espaces aquatiques. Ils imposent des règlementations qui constituent en fait des moyens de pressions. Les principes établis sont respectés, même s’ils sont dans certaines localités à l'origine des conflits. Ils sont suivis car les autorités villageoises sont plus proches des acteurs, dominés par les Maliens (54,74\%), Ghanéens (22,16\%) contre 19,25\% d'ivoiriens (Aka, 2012).

Pour les pêcheurs allogènes, le mode d'accès à la terre pour bâtir leur logis ou leur village obéit à des principes. Il faut obtenir au préalable une permission des autorités villageoises, suivie d'une petite libation, courroie de transmission entre les vivants et les morts. Toujours est-il que les locataires du nouveau village sont soumis à la règlementation du village-mère dont ils dépendent, l'enjeu étant important. Ce mode de gestion n'est pas spécifique au fleuve Comoé. Pliya (1985) en fait mention dans ses écrits sur les plans d'eau au Bénin, soulignant l'importance de la règlementation traditionnelle. Ainsi,"l'eau donne une assise et une influence à celui qui la possède" (Anoh, 2006). En somme, le mode de gestion traditionnelle est communément admis sur le Comoé et les riverains y sont attachés. Cependant, il n’exempte pas cet espace de diverses contraintes parmi lesquelles les conflits halieutiques figurent en bonne place.

\section{II/ Types de conflits, causes et moyens de leur reglement}

Les principales causes des conflits proviennent du paiement de la redevance villageoise, liant les autochtones aux allogènes et à un degré moindre aux allochtones. Á ce facteur se greffent les morts par noyade, les pratiques rituelles des pêcheurs maliens et d'autres actes antagoniques.

\section{1-Les conflits dus à la remise en cause de la redevance villageoise.}

La redevance est fondamentale dans la gestion des activités de pêche. Elle fait partie des clauses de l'installation des pêcheurs non-ivoiriens dans la quasi-totalité des villages riverains du Comoé. C'est une somme d'argent exigée chaque mois, de chacun de ces acteurs par la communauté villageoise qui l'accueille. Le non-respect des clauses établies aboutit inexorablement à des conflits.

En 2008, un conflit de ce type a opposé M’basso Agni et M’basso Attié, deux villages séparés par le Comoé et appartenant respectivement aux Sous-préfecture de Zaranou et d'Akoupé. Le conflit est survenu suite à une augmentation du montant de la redevance, passée de 1000 à 4000 Fcfa à 
M’basso Attié. En effet, cette situation a provoqué le déplacement des pêcheurs allogènes installés dans cette localité vers M’basso Agni. Les Attié s'en sont alors pris aux pêcheurs allogènes et aux Agni pour leur avoir donné asile. Cette attaque a occasionné de nombreux dégâts humains et matériels. Au plan humain, 12 blessés graves ont été enregistrés dont 6 pêcheurs de nationalité malienne, 4 ressortissants de M’Basso Attié et 2 de M’Basso Agni. On dénombre également 8 pirogues et des pagaies détruites, des filets endommagés ou emportés. L'apaisement a nécessité l'intervention pendant une semaine des brigades de gendarmerie d'Akoupé et de Zaranou.

La résolution de ce conflit a occasionné une rencontre à Zaranou à laquelle étaient conviés, outre les deux sous-préfets ayant la gestion des localités concernées, les chefs traditionnels Agni et Attié, les chefs des pêcheurs et les différents commandants de brigade. Les chefs des deux villages ont été mis en cause et reconnus comme responsables de cet affrontement pour n'avoir pas trouvé une solution préventive. Un compromis a par la suite été trouvé, celui de lever une redevance substantielle de 1500 FCFA/pêcheur/mois dans ces deux villages. Aussi, une prise en charge temporaire des blessés a-t-elle été évoquée.

\section{2-Le conflit issu de mort par noyade.}

Pendant la période de crue, les morts par noyade sont fréquents chaque année à Bassam et ses environs. Un jeune Abouré de Moossou retrouvé mort dans le filet des Bozo serait à l'origine d'un conflit entre ces deux entités en 2009. Le peuple Abouré les aurait accusés de l'avoir tué pour des pratiques occultes annuelles. Les autorités saisies de l'affaire ont procédé à une autopsie qui a confirmé un assassinat au lieu d’une noyade. Á l'annonce du rapport médical, la réaction des Abouré a été spontanée et a obligé les pêcheurs Bozo à fuir les habitations.

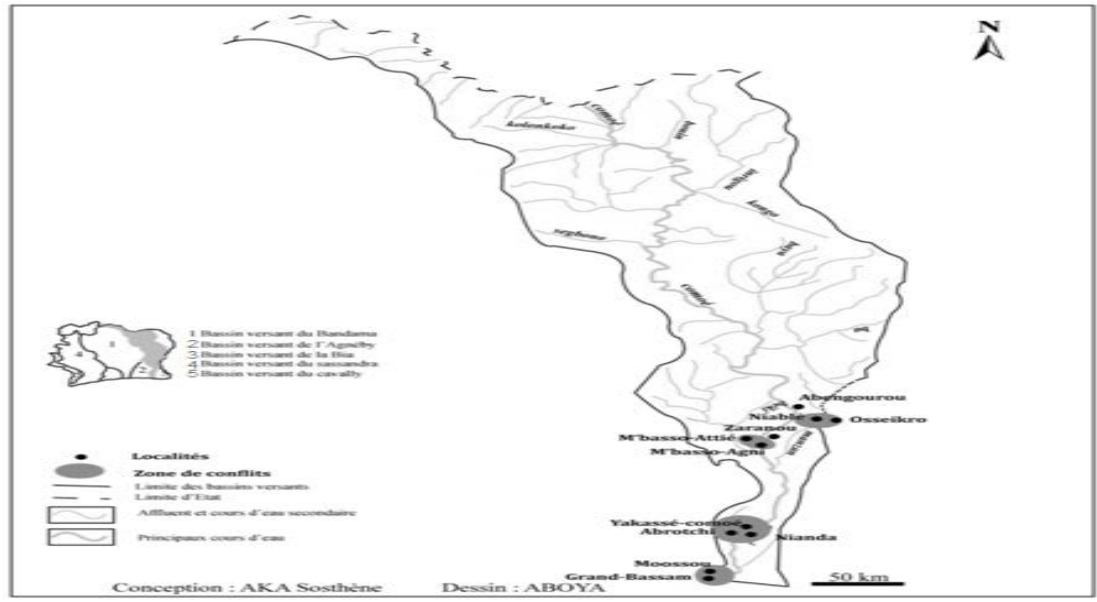

Figure 3 : Localisation des zones de conflits sur le bassin versant du Comoé. 
Tous les villages Abouré ont d'un commun accord, par la suite décidé de l'expulsion des Bozo de leurs eaux (fleuve Comoé et Lagune Ebrié) pour des attitudes incommodes. Pour qu'il y ait une accalmie, les autorités administratives ont ordonné aux responsables des pêcheurs Bozo de se retirer des eaux au vu de la tension, afin de leur permettre de trouver une solution définitive. Ce conflit a eu des répercussions jusqu'en pays Attié (Alépé). C'est la raison majeure pour laquelle les Bozo sont absents du Comoé dans l'espace Moossou-Alépé. C'est encore un autre conflit en latence. Ce comportement semble être l'apanage des pêcheurs maliens pour une abondance des captures, car ce sont les mêmes raisons qui ont valu leur expulsion du lac Ayamé en 1998. "Chaque année, à la remontée des eaux, les autochtones meurent par noyade. Cela est l'une des principales sources de conflits entre les populations autochtones riveraines et étrangères sur ce lac" (Vanga, 2001, p.163) qui a d'ailleurs connu un rebondissement pour les mêmes pratiques et fétichismes en janvier 2012 (ONG-PDHRE-CI ${ }^{(1)}, 2012$, p.44). Les espaces de tension sont précisés par la figure 3 ci-dessus.

\section{3-Autres types de conflits.}

\section{3-1-Les conflits nés de l'usage de produits toxiques.}

Le Manzan est l'un des importants affluents de la rive droite du Comoé et marque dans l'espace Niablé, la limite d'État, support frontalier entre la Côte d'Ivoire et le Ghana sur une distance d'environ $10 \mathrm{~km}$. Cet affluent a enregistré un conflit au mois d'août 1998. Du fait de sa localisation géographique, il est impérieux de soulever cet aspect pour permettre aux décideurs de trouver une solution définitive. Les peuples Agni de Niablé en Côte d'Ivoire et Ashante d'Osséikro au Ghana sont des agriculteurs dans cet espace. Cependant, en période de crue de la rivière Manzan, les employés des plantations (manœuvres) s'adonnent à l'activité de pêche. Une équipe de pêcheurs composée de burkinabé résidant au Ghana et quelques Awlan a empoisonné le Manzan à l'aide de produits toxiques agricoles dénommés $\mathrm{D}_{6}$ (Deltamétrine), un insecticide. Cette pratique a endommagé la faune aquatique (poissons, écrevisses,...) sur un grand espace. Informé, le chef canton de Niablé a fait arrêter et molester certains coupables au nombre de 6 . Il a tenu à avertir le Sous-préfet d'alors de Niablé, ainsi que les autorités coutumières et le représentant de l'administration d'Osséikro. Après constat et concertations, les autorités ont laissé la latitude aux chefs coutumiers frontaliers de cet espace dont la sagesse les a conduits à une résolution pacifique et durable.

${ }^{(1)}$ PDHRE-CI = People's movement for Human Right Education Côte d'Ivoire (Mouvement des Peuples pour l'Education aux Droits Humains de Côte d'Ivoire). 
Les frontières d'État étant des zones sensibles, il convient de prendre des mesures adéquates pour éviter les énormes dégâts. Toutes les oppositions d'intérêts dans un tel espace méritent une attention soutenue et doivent être traitées avec plus d'égard.

\section{3-2-Les conflits liés aux vols de captures.}

Ce type de conflit s'est produit en 2010 entre 3 villages riverains du Comoé de la Sous-Préfecture d'Alépé que sont Nianda, Yakassé-Comoé et Abrotchi indiqués par la figure 4. Yakassé-Comoé et Nianda sont propriétaires de l'espace aquatique selon le découpage ancestral, qui demeure en vigueur aujourd'hui. Par principe de bon voisinage, YakasséComoé a octroyé une partie de son espace fluvial à Abrotchi parce que cette localité est située à $1,5 \mathrm{~km}$ environ du fleuve. Ainsi les trois villages participent à la pêche en partageant le même espace aquatique. cependant, en 2010, des actes de vols de poissons et d'écrevisses commis à l'égard des deux autres localités par des jeunes pêcheurs d'Abrotchi ont mis à mal cette cohabitation pacifique. Pris sur les faits, les mis en cause ont été battus. Il s'en suivit une bataille rangée entre les jeunes d'Abrotchi et ceux des deux autres villages. Ce conflit a fait réapparaitre la question des terroirs halieutiques octroyés à Abrotchi et leur interdiction aux pêcheurs dudit village.

Cette situation a suscité l'intervention des autorités administratives de la région (Préfet et Sous-préfet) pour la recherche d'une solution. La décision a contraint les populations de chaque village à pratiquer la pêche uniquement dans leur espace respectif. Une solution est trouvée par les responsables de villages en se référant aux clauses traditionnelles et aux sanctions antérieures. Chaque coupable a une amende de 50000 FCFA à verser à la caisse associative des autres villages avec une interdiction de pêche dans l'espace Comoé sur une période de 5 ans pour le village d'Abrotchi.

Sur le Comoé, contrairement aux conflits engendrés par les contestations des engins de pêche, ce sont ceux des sacrifices rituels, de redevances ou de vols de captures. Le seul conflit meurtrier est celui de Moossou. Une résolution locale régie par les institutions traditionnelles a toujours prévalu. En somme, les tensions naissent de l'application des lois coutumières et des mauvaises pratiques de pêche. Cependant, la pression qui s'exerce sur la faune aquatique invite à la recherche de mesures alternatives susceptibles de mieux la juguler. 


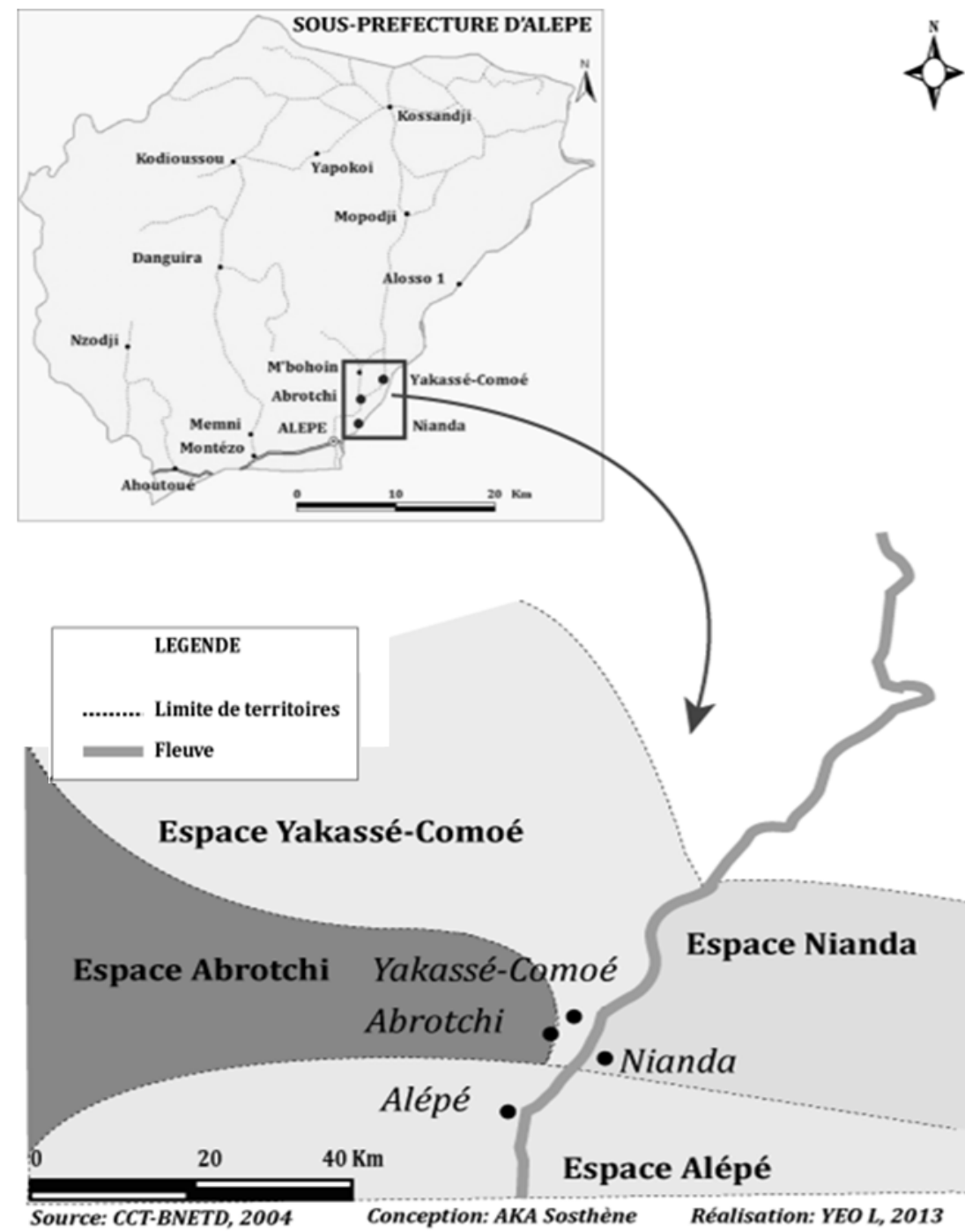

Figure 4 : Délimitation traditionnelle des territoires entre Nianda, Abrotchi et YakasséComoé.

III/ Analyse des moyens de reglement des conflits et esquisse de solutions pour une gestion durable de la peche sur le comoe et ses affluents

\section{1-Analyse des moyens de règlement des conflits}

Les activités de pêche sont indispensables, complexes et comprennent des mécanismes de gestion, des modes d'exploitation qui font ressortir la notion d'appartenance de territoires. Les autorités villageoises 
qui administrent la pêche sur le Comoé se doivent de régler les conflits. Á cet effet, elles y sont associées pour leur maîtrise de cet environnement. Les causes de ces tensions peuvent être lointaines, correspondant dans de tels cas à l'accumulation de redevances impayées, à la récurrence des morts par noyade ou des vols des captures. Les moyens de règlement de ces conflits sont efficaces parce qu'ils reposent sur un consensus de toutes les parties prenantes. Il s'agit des préfets et sous-préfets en tant que gestionnaires du territoire concerné et représentant de l'administration centrale, des chefs traditionnels en qualité de gestionnaires de l'espace auquel ils appliquent les lois coutumières. En amont de l'activité, l'on note la présence des pêcheurs, responsables de la majeure partie des conflits. Á cette liste s'ajoutent les forces de l'ordre, chargées d'assurer la sécurité des hommes et des biens. Au niveau du Comoé, les chefs coutumiers sont d'un apport essentiel dans la gestion et le règlement des conflits. Les préfets et sous-préfets sont quasiabsents des espaces de pêche, mais remplissent leur rôle d'autorité en cas de nécessité.

Cependant, des insuffisances demeurent et se caractérisent par un manque de confiance et une instabilité dans les institutions coutumières. L'eau appartenant au domaine public, les conflits doivent trouver leurs solutions par la mise en pratique des textes et obéir à des méthodes. Á cet égard, tout semeur de trouble ou utilisateur de produits prohibés ou encore auteur de malversations doit subir la loi. La décision n08/MDR/12 mars 1984 par exemple, en son article 10 autorise les services de police, la gendarmerie et les eaux et forêts à constater les infractions et à appliquer les sanctions correspondantes. Quant à la loi nº97-766 du 3 octobre 1997, elle fait de l'environnement une priorité pour éviter sa dégradation sous toutes ses formes, prévoit la délivrance d'une licence de pêche dans le domaine artisanal. Elle renforce le contrôle de l'accès à l'eau et l'octroi d'une autorisation préalable, mentionne également les méthodes de pêche prohibées (produits toxiques, explosifs, armes à feu, électrocution) en ses articles 11 et 12. Malheureusement les services halieutiques sont quasi absents des résolutions de conflits. Pourtant, ce rôle de gestionnaire de l'eau leur est dévolu.

\section{2-Esquisse de solutions pour une gestion durable de la pêche sur le Comoé et ses affluents}

Pour éviter les tensions à répétition, avec des conséquences néfastes, force serait de privilégier le cadre juridique. Il faut donc insister sur l'appartenance du fleuve Comoé au domaine public, car "la mauvaise définition légale de la propriété est souvent à l'origine de multiples conflits entre pêcheurs et comité de gestion, parfois exacerbés par la nationalité des pêcheurs"(Da Costa et al. 1998, p.76). Ce fleuve manque d'un contrôle et 
d’une gestion véritablement étatique, faute d'une application correcte des textes légaux qui interdisent certaines pratiques et autorisent des sanctions pour les infractions commises (Loi n97-766 du 3 octobre 1997 et décision $\mathrm{n}^{\circ} 08 / \mathrm{MDR} / 12$ mars 1984). L’absence de cette rigueur est à l’origine de tensions."La gestion durable de la question foncière (ainsi que les plans d'eau rattachés) requiert une ferme volonté politique pour mener à bien des réformes"(Ntampaka, 2008, p.18). Pour sa mise en œuvre, il faut une sensibilisation à la légalisation de l'activité par les opérateurs économiques, les acteurs du Comoé vivant dans l'informel. L’obtention d'une licence de pêche doit constituer un préalable pour exercer véritablement le métier de pêcheur. Etant identifiés, leur gestion peut être aisée. Il faut également une forte implication de l'État, de tous les autres acteurs dans la gestion des eaux et des conflits. L'urgence est "d'élaborer des programmes d'information, de sensibilisation et d'éducation des utilisateurs en rapport avec l'objectif des législations visant à améliorer les comportements et les relations entre les différents acteurs" (Adam, 2008, p.8). Seul le Ministère des Ressources Animales et Halieutiques est habilité à mieux la structurer. La pénurie des agents des ressources halieutiques est criarde avec un effectif de 34 agents pour gérer $900 \mathrm{~km}$ de fleuve (Aka, 2012). L'essentiel pour la tutelle serait de renforcer la capacité humaine par la formation et de procéder à leur bonne répartition dans l'espace ivoirien en fonction des besoins avec des missions précises. Ils doivent être appuyés dans l'exercice de leur fonction par les forces de l'ordre. Cela peut servir de prévention à certains conflits. Aussi, ne serait-il pas utile de calquer sur le modèle ANADER (Agence Nationale d’Appui au Développement Rural) dans sa politique de rapprochement de ses agents des populations agricoles ? Il faut à cet effet créer des relations plus étroites entre les agents halieutiques et les utilisateurs de l'eau, tout en leur donnant des moyens de locomotion. Ce facteur peut aider à harmoniser les conditions d'accès au Comoé par des textes et des lois coutumières. Le mode d'accès à l'eau par les riverains n’est pas identique."Les modalités d'appropriation et d'accès au terroir amphibie sont variables selon les acteurs, les formes d'exploitation et la saison" (Cormier-Salem, 2000, p.216).

Il est aussi primordial d'adopter un plan d'aménagement concerté en Côte d'Ivoire comme l'entrevoit l’UEMOA (Union Économique Monétaire Ouest-Africaine) dont l'objectif du programme est de "proposer un cadre juridique communautaire d'exploitation, de gestion et de contrôle des activités de la pêche dans les eaux continentales des Etats membres" (UEMOA, Avril 2007, p.11). La cogestion peut être un outil efficace pour éviter les conflits. C'est une forme d'administration qui implique tous les acteurs sans faire tort à un groupe. Elle a été expérimentée au Ghana et décrite à travers une étude menée par Sanogo (2002) qui la définit en tant 
"qu'un accord de partenariat dans lequel les agents de l'État, la communauté des utilisateurs locale (pêcheurs), les organisations non gouvernementales (ONG) et autres partenaires partagent la responsabilité et l'autorité dans la gestion de la pêcherie". Au niveau des eaux intérieures, on peut chercher à réduire la pression sur le stock à travers l'encouragement ou l'exécution de grands travaux par l'État dans le domaine halieutique. Au niveau du Comoé, ce sont des quantités d'eau démesurées qui coulent chaque année, surtout en période de crue. Par des stratégies d'aménagement (système de canalisation et autres), des travaux piscicoles peuvent être exécutés.

\section{Conclusion}

Au terme de ce travail, l’on peut retenir que le Comoé, support des activités halieutiques connaît une pêche artisanale et les riverains en tirent profit à partir d'une double perception au plan économique et culturel. Considéré comme leur propriété, ce fleuve présente des foyers de tensions nés de l'application des lois coutumières et des mauvaises pratiques des acteurs. Les conflits ne sont pas d'une grande ampleur à l'exception de celui de Moossou qui s'est étendu au-delà d'un rayon de $50 \mathrm{~km}$ (Moossou à Alépé). Ils connaissent des dénouements heureux et les moyens de leur règlement sont efficaces parce que souvent issus d'un accord auquel les autorités administratives, les autorités coutumières et les pêcheurs y prennent une part active. Cependant l'application rigoureuse des textes, à défaut d'une mise en place d'un système de cogestion peut encore rendre très crédibles les résolutions et épargner au mieux cet espace des oppositions d'intérêts. Aussi, le Comoé peut-il faire l'objet d'une politique de gestion stricte associant les textes de loi et les pratiques coutumières pour éviter trop de heurts. Ce thème mérite une attention particulière et doit connaître un approfondissement pour épargner des vies humaines et éviter les différentes perturbations de la chaîne halieutique.

\section{References:}

1. Adam M. K.,(2008). Gouvernance foncière et des ressources naturelles ; état des lieux en Afrique de l'Ouest, Rome, FAO, 39 p.

2. Aka K. S.,(2012). Le fleuve Comoé et l'organisation de l'espace halieutique, thèse unique de Doctorat, univ. Cocody Abidjan, Côte d'Ivoire, 307 p.

3. Anoh K. P.,(2006). Patrimoine aquatique et exploitation des plans d'eau du littoral ivoirien : le cas des lagunes de Grand-Lahou (Côte d'Ivoire) in Chaussade J., Guillaume J., pêche et aquaculture pour une exploitation durable des ressources vivantes de la mer et du littoral, Rennes, PUR, pp. 443-459. 
4. Brunet R., Ferras R., Thery H.,(1992). Les mots de la géographie, Dictionnaire critique, Paris, Reclus-la documentation française.

5. Cormier-Salem M.C.,(2000) Appropriation des ressources, enjeu foncier et espace halieutique sur le littoral Ouest-africain in Les pêches piroguières en Afrique de l'Ouest: pouvoirs, mobilités, marchés, Edition Karthala, CMI et IRD, pp. 205-229.

6. Da Costa K. S., Traoré K., Tito de Morais L.,(1998). Effort de pêche et production exploitée dans les petites retenues du Nord de la Côte d'Ivoire. Bull.fran.pêche.piscic.71 (348) pp. 65-78.

7. FAO,(2008). Vue générale du secteur des pêches national; la République de Côte d'Ivoire, FAO, Rome, 43 p.

8. Merlet M.,(2011). Points chauds liés au foncier et au droit sur l'eau in solaw background thematic report-TRO5, AGTER Paolo Groppo, FAO, 34 p.

9. Norem R. H.,(1997). Une démarche conceptuelle pour la planification, la mise en ouvre, le suivi et l'évaluation du développement ; analyse socio-économique selon le genre. Document français préparé à partir de la version anglaise ; FAO/OIT, 116 p.

10. Ntampaka C.,(2008). Gouvernance foncière en Afrique Centrale, FAO, Rome, 59 p.

11. ONG P.D.H.R.E. et H.A.C.,(2012). Rapport Ayamé - Conflits halieutiques, Abidjan Côte d'Ivoire, 44 p.

12. Pliya J.,(1985). Protection du milieu et législation traditionnelle de protection en Afrique de l'Ouest, Communication au colloque de Cotonou, Déc. 1985, 10 p.

13. Sanogo D. A.,(2002). La cogestion comme une option pour une gestion durable de la pêche artisanale en Afrique Subsaharienne : le cas du Ghana ; Rapport de recherche, Univ. du Québec, 55 p.

14. UEMOA,(2007). Plan d'aménagement concerté des pêches et d'aquaculture au sein de l'UEMOA; Programme d'action, Avril 2007, 51 p.

15. Vanga A. F.,(2001). Conséquences socio-économiques de la gestion des ressources naturelles : cas des pêcheries dans les lacs d'Ayamé et de Buyo (Côte d'Ivoire), thèse de Doctorat de l'univ. d'AboboAdjamé, Abidjan Côte d'Ivoire, 210 p.

16. Yao S., Essetchi P.K., Koné J., N’Douba V., Gooré Bi G., Frans O. Thys Van Den Audenaerde,(2005). Fish communities along environmental gradients within the Comoé river bassin, Côte d'Ivoire, in African journal of aquatic science, pp. 185-194. 